Accepted By The Astrophysical Journal

Preprint typeset using $\mathrm{LAT}_{\mathrm{E}} \mathrm{X}$ style emulateapj v. 5/2/11

\title{
SYNCHROTRON SPECTRAL CURVATURE FROM 22 MHZ TO 23 GHZ
}

\author{
A. KoGUT $^{1}$ \\ Accepted by The Astrophysical Journal
}

\begin{abstract}
We combine surveys of the radio sky at frequencies $22 \mathrm{MHz}$ to $1.4 \mathrm{GHz}$ with data from the ARCADE2 instrument at frequencies 3 to $10 \mathrm{GHz}$ to characterize the frequency spectrum of diffuse synchrotron emission in the Galaxy. The radio spectrum steepens with frequency from $22 \mathrm{MHz}$ to $10 \mathrm{GHz}$. The projected spectral index at $23 \mathrm{GHz}$ derived from the low-frequency data agrees well with independent measurements using only data at frequencies $23 \mathrm{GHz}$ and above. Comparing the spectral index at $23 \mathrm{GHz}$ to the value from previously published analyses allows extension of the model to higher frequencies. The combined data are consistent with a power-law index $\beta=-2.64 \pm 0.03$ at 0.31 $\mathrm{GHz}$, steepening by an amount $\Delta \beta=0.07$ every octave in frequency. Comparison of the radio data to models including the cosmic ray energy spectrum suggests that any break in the synchrotron spectrum must occur at frequencies above $23 \mathrm{GHz}$.
\end{abstract}

Subject headings: radio continuum: general, radiation mechanisms: non-thermal

\section{INTRODUCTION}

Synchrotron emission from relativistic cosmic ray electrons accelerated in the Galactic magnetic field dominates the diffuse radio continuum at frequencies below $1 \mathrm{GHz}$. It is an important foreground contaminant for measurements of the cosmic microwave background radiation, and also serves to probe the Galactic magnetic field and cosmic ray distributions. Measurements of the synchrotron frequency spectrum are thus of interest to several areas in astrophysics.

An isotropic distribution of relativistic electrons at a single energy $E=\gamma m c^{2}$ propagating in a uniform magnetic field $B$ has emissivity

$$
\epsilon(\nu)=\frac{\sqrt{3} e^{3}}{m c^{2}} B \sin \alpha F(x),
$$

where $\alpha$ is the pitch angle between the magnetic field and the line of sight, and

$$
F(x)=x \int_{x}^{\infty} K_{5 / 3}\left(x^{\prime}\right) d x^{\prime}
$$

is defined in terms of the modified Bessel function of order $5 / 3$ with variable $x=\nu / \nu_{c}$ and

$$
\nu_{c}=\frac{3}{4 \pi} \frac{e}{m c} \gamma^{2} B \sin \alpha
$$

(Schwinger 1949; Westfold et al. 1959; Oster 1961). For a power-law distribution of electron energies $N(E) \propto E^{p}$ propagating in a uniform magnetic field, the synchrotron emission is also a power law,

$$
T_{A}(\nu) \propto \nu^{\beta}
$$

where $T_{A}$ is antenna temperature, $\nu$ is the radiation frequency, and

$$
\beta=\frac{p-3}{2}
$$

Alan.J.Kogut@nasa.gov

${ }^{1}$ Code 665, Goddard Space Flight Center, Greenbelt, MD 20771
(Rybicki \& Lightman 1979).

Measurements of the synchrotron spectral index provide important input for models of cosmic ray propagation. Solar modulation reduces the local cosmic ray electron density for electron energies below a few $\mathrm{GeV}$ so that synchrotron emission provides the most direct probe of low-energy cosmic rays. Measurements of the cosmic ray spectrum above a few $\mathrm{GeV}$ in turn inform models of the high-frequency synchrotron spectrum. Energy losses from cosmic ray propagation steepen the cosmic ray spectrum, increasing $p$ toward higher energies. The observed steepening from $p \sim-2.6$ at $5 \mathrm{GeV}$ to $p \sim-3.2$ at $50 \mathrm{GeV}$ predicts a corresponding steepening in the synchrotron spectrum from $\beta \sim-2.8$ at $1 \mathrm{GHz}$ to $\beta \sim-3.1$ at $100 \mathrm{GHz}$ (Strong, Moskalenko, \& Ptuskin 2007).

Comparison of the cosmic ray spectra to the predicted synchrotron spectrum is complicated by confusion from competing radio emission sources. The diffuse radio continuum is a superposition of the cosmic microwave background, synchrotron emission, free-free emission from the warm ionized interstellar medium, and emission from interstellar dust. A number of authors have attempted to disentangle the various emission sources to determine the synchrotron spectral index (for a recent review see Appendix A of Strong, Orlando, \& Jaffe (2011)). Despite some discrepant results, the general trend shows a steepening of the synchrotron spectrum from $\beta \sim-2.5$ at 22 $\mathrm{MHz}$ to $\beta \sim-3.0$ above $23 \mathrm{GHz}$, in rough agreement with the observed cosmic ray spectra.

Several factors contribute to the observed scatter in estimates of the synchrotron spectral index. Most estimates, particularly those below $23 \mathrm{GHz}$, assume a power-law spectrum for synchrotron and do not explicitly model spectral steepening. Comparisons between closely-separated frequencies more accurately reflect the local synchrotron spectrum, but have larger uncertainties from competing emission sources or measurement offsets. Analyses with broader frequency coverage reduce foreground and offset uncertainties but average over any spectral steepening.

Two additional effects are important for analy- 
ses including data from the Wilkinson Microwave Anisotropy Probe (WMAP) at frequencies 23 to 94 $\mathrm{GHz}$. A growing body of evidence suggests that a substantial fraction of the diffuse continuum near $23 \mathrm{GHz}$ consists of electric dipole radiation from a population of small, rapidly spinning dust grains (Kogut et al. 1996; de Oliveira-Costa et al. 1997, 2004; Miville-Deschênes et al. 2008; Dobler \& Finkbeiner 2008; $\quad$ Ysard, Miville-Deschênes, \& Verstraete 2010; Kogut et al. 2011; Gold et al. 2011; Planck collaboration 2011). Spinning dust emission is expected to peak at frequencies near $20 \mathrm{GHz}$ (Draine \& Lazarian 1998; Ali-Haïmoud et al. 2009; Hoang, Draine, \& Lazarian 2010; Ysard \& Verstraete 2010). Analyses that ignore this component to attribute the observed emission only to synchrotron radiation tend to over-predict the synchrotron amplitude at frequencies near $23 \mathrm{GHz}$, biasing the derived spectral index to flatter values when comparing to lower frequencies and steeper values when comparing to higher frequencies.

A second systematic error can result from improper treatment of offsets in the data. Measurements from radio surveys at frequencies below $20 \mathrm{GHz}$ generally include the absolute intensity (zero level) of the sky. The WMAP differential radiometers are insensitive to any constant (monopole) intensity on the sky; the zero level of the WMAP sky maps is set so that the map intensity in the Galactic polar caps matches a cosecant fit to the midlatitude sky (Bennett et al. 2003; Hinshaw et al. 2009). Analyses that directly compare low-frequency radio surveys to the WMAP data without subtracting a monopole component from the radio data will miss the corresponding emission in the WMAP bands, biasing the derived spectral index to steeper values.

The zero level bias can be significant. The Haslam et al. (1981) survey at $408 \mathrm{MHz}$ is commonly used to model synchrotron emission. The North Galactic pole has measured temperature $19 \pm 3 \mathrm{~K}$ at $408 \mathrm{MHz}$, while a csc $|b|$ fit to the same $408 \mathrm{MHz}$ map predicts a polar contribution of only $5.1 \pm 0.6 \mathrm{~K}$. Similar results

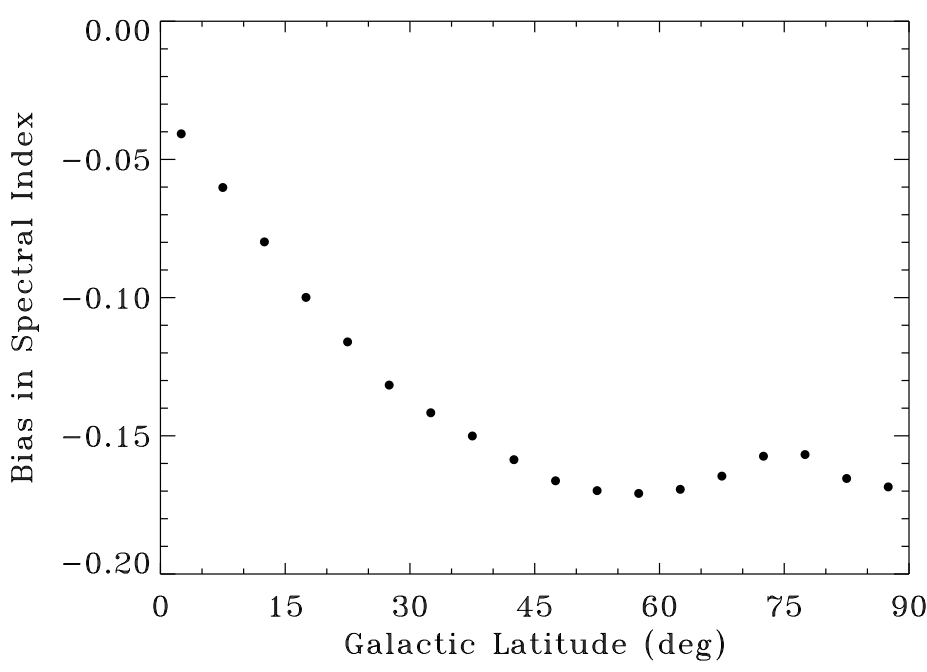

FIG. 1.- Toy model showing effect of improper zero level subtraction on the derived spectral index between $408 \mathrm{MHz}$ and 23 $\mathrm{GHz}$.
TABLE 1

Sky Surveys Used FOR SynChrotron ANALYSIS

\begin{tabular}{cccc}
$\begin{array}{c}\text { Frequency } \\
(\mathrm{GHz})\end{array}$ & $\begin{array}{c}\text { Calibration } \\
\text { Uncertainty }\end{array}$ & $\begin{array}{c}\text { Offset } \\
\text { Uncertainty }(\mathrm{K})\end{array}$ & $\begin{array}{c}\text { Relative } \\
\text { Uncertainty }^{a}\end{array}$ \\
\hline 0.022 & 0.05 & 5000 & 0.15 \\
0.045 & 0.10 & 250 & 0.11 \\
0.408 & 0.10 & 3.0 & 0.17 \\
1.420 & 0.05 & 0.5 & 0.63 \\
3.20 & 0.001 & 0.011 & 0.10 \\
3.41 & 0.001 & 0.006 & 0.07 \\
7.98 & 0.001 & 0.036 & 0.89 \\
8.33 & 0.001 & 0.042 & 2.64 \\
9.72 & 0.001 & 0.003 & 0.34 \\
10.49 & 0.001 & 0.002 & 0.27 \\
\hline${ }^{a}$ Quadrature sum of calibration and offset uncertainties,
\end{tabular}

divided by the mid-latitude sky temperature.

apply to the Southern hemisphere, where the measured polar cap temperature of $21 \pm 3 \mathrm{~K}$ significantly exceeds the value $4.0 \pm 0.5 \mathrm{~K}$ obtained from a csc $|b|$ fit. Only 2.7 $\mathrm{K}$ of the difference can be attributed to emission from the cosmic microwave background, leaving a large residual.

Figure 1 illustrates the bias induced by including this residual at $408 \mathrm{MHz}$ but excluding it from the WMAP data. We take the $408 \mathrm{MHz}$ map, remove the $2.7 \mathrm{~K}$ $\mathrm{CMB}$ monopole, and scale the remaining (radio) emission to $23 \mathrm{GHz}$ using a power-law index $\beta=-2.7$ to produce a toy model of the radio sky at $23 \mathrm{GHz}$. Following the WMAP processing, we then remove a monopole from the scaled map so that the map temperature in the south polar cap matches the csc $|b|$ fit. We then compute the bias in apparent spectral index by comparing the $408 \mathrm{MHz}$ map to the scaled $23 \mathrm{GHz}$ map before and after removing the scaled monopole. Figure 1] shows the bias in spectral index at $23 \mathrm{GHz}$, binned by Galactic latitude. Dis-similar treatment of the map zero level creates a spatially varying bias $\Delta \beta \approx 0.15$, comparable to the total spectral steepening predicted by the measured cosmic ray spectra. The bias is largest in regions where the sky brightness is faintest, at high latitudes or away from the Galactic center.

Measurement uncertainties in the absolute level of the sky brightness can also introduce bias in estimates of the synchrotron spectral index. Many of the low-frequency surveys have uncertainty in the measured zero level approaching $30 \%$ of the polar cap brightness. As with the toy model above, such measurement errors introduce spatially dependent biases that are largest where the sky brightness is faintest. Minimizing uncertainty in the derived synchrotron spectrum requires a combination of measurements with good sky coverage and good control of offset uncertainty at frequencies where competing emission sources are faint. No such ideal data set yet exists. In this paper, we model the synchrotron spectral index and curvature using low-frequency radio surveys with high sky coverage but large offset uncertainty, combined with higher-frequency measurements with limited sky coverage but still useful offset uncertainty.

\section{SKY MAPS}

We model synchrotron emission using radio surveys at $22 \mathrm{MHz}$ (Roger et al. 1999), $45 \mathrm{MHz}$ (Maeda et al. 1999; Alvarez et al. 1997), $408 \mathrm{MHz}$ (Haslam et al. 1981), and $1420 \mathrm{MHz}$ (Reich. Testori, \& Reich 2001; Reich \& Reich 1986). These surveys have full or nearlyfull sky coverage at frequencies where Galactic radio 


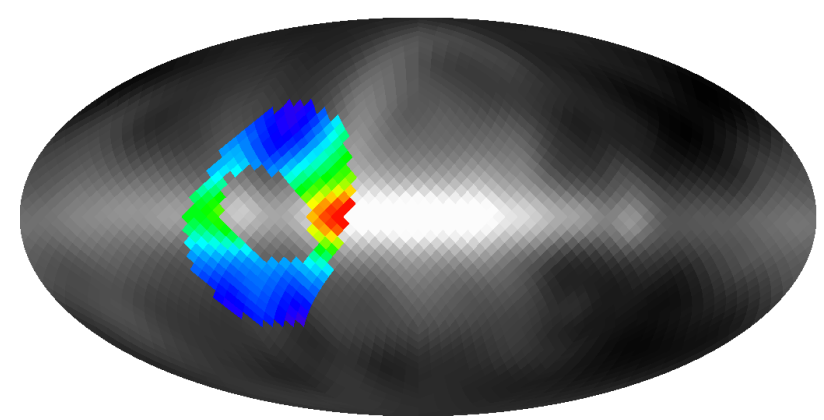

FIG. 2.- Sky coverage for this analysis. The plot shows the 408 $\mathrm{MHz}$ sky survey convolved to $11^{\circ} 6$ angular resolution. Pixels common to all 10 radio surveys are shown in color. The sky coverage is limited by the ARCADE 2 observations but includes the Galactic plane, mid-latitude sky, and portions of the North Galactic Spur (radio Loop I).

emission is significant, with gain and zero-level systematics controlled at the 10-20\% level. We supplement the radio surveys with sky maps from the Absolute Radiometer for Cosmology, Astrophysics, and Diffuse Emission (ARCADE 2) instrument2 at 3, 8, and $10 \mathrm{GHz}$ Kogut et al. 2011). The ARCADE 2 data observe both the Galactic plane and mid-latitude regions $\left(|b|<40^{\circ}\right)$ with sufficient control of zero-level uncertainty to constrain the synchrotron curvature relative to the lower-frequency radio surveys.

Table 1 summarizes the input sky maps. The increase in the offset uncertainty at low frequency is compensated by a corresponding increase in sky brightness. The final column shows the relative measurement uncertainty for a mid-latitude region, defined as the ratio of the combined offset and calibration uncertainty to the measured brightness at $(l, b)=\left(17^{\circ},-35^{\circ}\right)$ after removing the CMB monopole. The selected maps provide roughly uniform relative sensitivity to synchrotron emission over 2.5 decades of frequency.

We convert all maps to units of antenna temperature and subtract the CMB monopole at (thermodynamic) temperature $2.725 \mathrm{~K}$ from the measured sky temperatures. We then convolve each map to the $11: 6$ angular resolution of the ARCADE 2 instrument. At frequencies of $10 \mathrm{GHz}$ and below, both thermal dust emission and spinning dust emission are negligible. Free-free emission, however, can still be appreciable. We correct the convolved maps by scaling the WMAP 7-year maximum entropy free-free model (Gold et al. 2011) to each frequency using spectral index -2.15 , convolving the scaled model to 11.6 angular resolution, and subtracting the convolved model from each sky survey. The resulting maps are dominated by synchrotron emission.

\section{ANALYSIS}

2 The ARCADE data are available at the Legacy Archive for Microwave Background Data Analysis, http://lambda.gsfc.nasa.gov
The input sky maps define a data set $T(\hat{n}, \nu)$ sampled at discrete pixel directions $\hat{n}$ and 10 discrete frequencies $\nu$ ranging from $22 \mathrm{MHz}$ to $10 \mathrm{GHz}$. We restrict the analysis to the $8 \%$ of the sky observed at all 10 frequencies. Figure 2 shows the resulting sky coverage. Within the common sky coverage, we model synchrotron emission as a modified power law

$$
T(\hat{n}, \nu)=A(\hat{n})\left(\frac{\nu}{\nu_{0}}\right)^{\beta+C \ln \left(\nu / \nu_{0}\right)}
$$

with spectral index $\beta$ and curvature $C$ defined with respect to reference frequency $\nu_{0}=310 \mathrm{MHz}$. The adopted value for $\nu_{0}$ minimizes covariance between the fitted amplitude $A$ and spectral index $\beta$, simplifying extrapolation to other frequencies.

For each pixel $\hat{n}$ we define a 10 by 10 data covariance matrix $M$ with diagonal elements determined by the instrument noise, calibration and offset uncertainty. The input maps are not all linearly independent. Measurements at $22 \mathrm{MHz}$ used the $408 \mathrm{MHz}$ map to determine the declination dependence of the gain. We estimate the resulting correlation of spatial structure in the two maps at 50\%. The ARCADE 2 maps have independent instrument noise but share a fraction of the offset uncertainty related to absolute thermometry uncertainty and ground glint (Singal et al. 2011). All 10 maps share a common model for free-free emission. We conservatively estimate the uncertainty in the free-free correction at $30 \%$ of the free-free amplitude; however, the results do not change significantly as the model free-free amplitude is varied by as much as $50 \%$. Off-diagonal elements in $M$ include these effects.

For each pixel, a least-squares minimization determines the best-fit parameters $A, \beta$, and $C$. Figure 3 shows the measured temperature and best-fit model for the brightest Galactic plane region $(l, b)=\left(52^{\circ}, 0^{\circ}\right)$ within the common sky coverage. The data show evidence for spectral curvature, with best-fit values $\beta=-2.60 \pm 0.04$ and

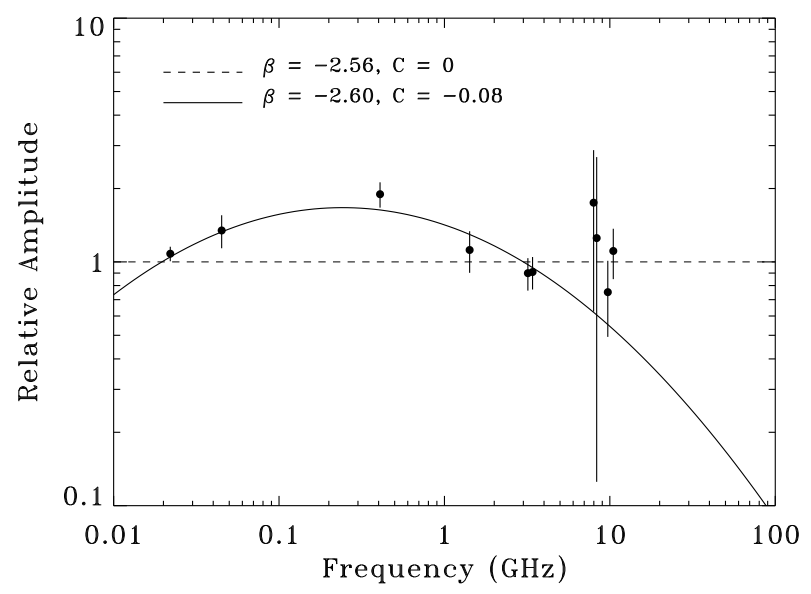

FIG. 3.- Sky temperatures and best-fit model (solid line) for a $4^{\circ}$ diameter patch on the Galactic plane centered on the the brightest pixels in the ARCADE 2 sky coverage. For clarity, the data are plotted relative to the best-fit power-law model $(\beta=-2.56)$ with zero curvature (dashed line). All fits include the non-trivial covariance between individual data points. The data are well described by a model with spectral index $\beta=-2.60 \pm 0.04$ and curvature $C=-0.081 \pm 0.028$. 


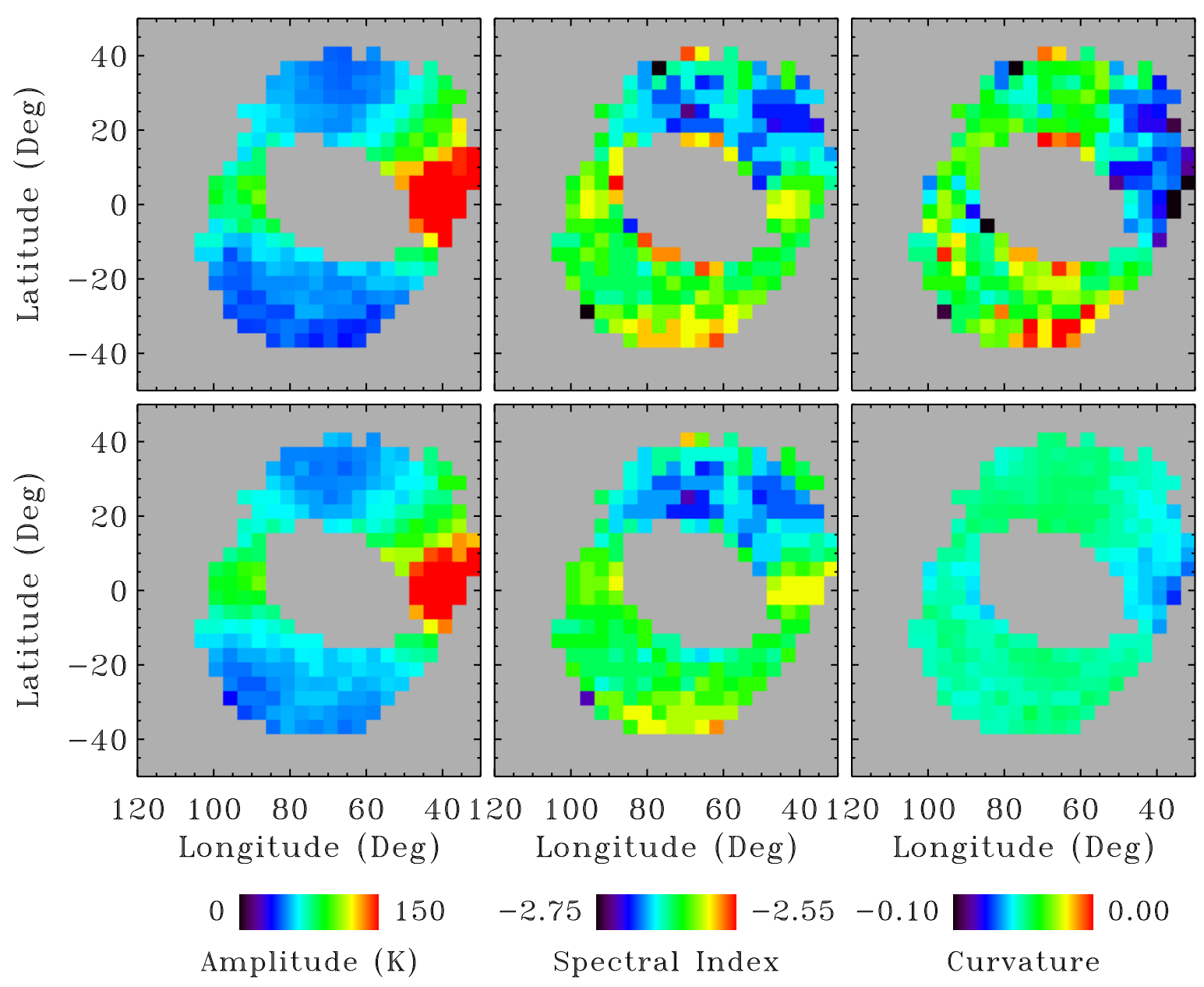

FIG. 4. - Sky maps of best-fit spectral parameters. Left to right: synchrotron amplitude, spectral index, and curvature evaluated at reference frequency $310 \mathrm{MHz}$. The top panels show results from the 10-frequency fit, while the bottom panels include the spectral constraint at $23 \mathrm{GHz}$.

$C=-0.081 \pm 0.028$ evaluated at $\nu_{0}=310 \mathrm{MHz}$. The spectral curvature $C$ for this region is significant at approximately 3 standard deviations compared to the baseline model with $C=0$.

We may extrapolate the spectral models to compare the results at frequencies $10 \mathrm{GHz}$ and below to independent determinations of the spectral index using WMAP data at frequencies $23 \mathrm{GHz}$ and above. We compute the antenna temperature of the modeled spectra to derive the effective power-law index for frequencies near 23 $\mathrm{GHz}$. Note that this is not equivalent to evaluating Eq. 6] at $\nu=23 \mathrm{GHz}$, which would yield the scaling from 310 $\mathrm{MHz}$ to $23 \mathrm{GHz}$ but not the power-law index at $23 \mathrm{GHz}$. The mean for all 258 pixels in the common sky coverage is $\beta_{23}=-3.02$ with standard deviation 0.22 .

The extrapolated value compares well with independent determinations of the spectral index above $23 \mathrm{GHz}$. Kogut et al. (2004) analyze WMAP polarization data to derive synchrotron spectral index $\beta=-3.2 \pm 0.1$ averaged over the full sky. Dunkley et al. (2009) use a Bayesian analysis of polarization data and find the mean spectral index $\beta=-3.03 \pm 0.04$ with pixel-topixel standard deviation 0.25 over the high-latitude sky. Gold et al. (2011) use template fitting techniques to derive spectral index $\beta=-3.13$ between 23 and $33 \mathrm{GHz}$.

The mean spectral index derived from the 10 low- frequency radio surveys agrees with the value derived from WMAP data at higher frequencies. Much of the scatter in the extrapolated spectral indices results from pixels at high latitude where the emission is faintest. The extrapolated index in these pixels can reach unphysical values. We reduce the scatter in the fitted spectral parameters by applying additional constraints using WMAP data at $23 \mathrm{GHz}$ and above. The simplest such constraint, adding WMAP temperature data to the multi-frequency fit, is problematic. Not only would the procedure need to include additional free parameters to account for emission from thermal dust or spinning dust (both negligible at lower frequencies), but each of the low-frequency maps would require a correction to remove the monopole contribution missing from the WMAP data. Although the WMAP zero level is clearly defined by a csc $|b|$ fit to mid-latitude data, the astrophysical interpretation of a similar procedure applied to low-frequency radio surveys is less clear. The coldest region of the radio sky is not at the Galactic poles, but at mid-latitudes above the Galactic anti-center. Subtraction of too large a monopole can leave unphysical negative residuals. Limited sky coverage exacerbates this problem.

We avoid these problems by using a constraint based on the spectral index derived solely from WMAP data. 
For each pixel, we use the radio data (Table 1) to fit the synchrotron amplitude $A(\hat{n})$ over a 2-dimensional grid in the spectral parameters $\beta$ and $C$ (Eq. 6). At each grid point, we compute the $\chi^{2}$ value $R^{T} M^{-1} R$ where $M^{-1}$ is the inverse covariance matrix and $R$ is the difference vector between the measured and modeled temperatures. We then use the spectral parameters $\beta$ and $C$ to evaluate the power-law index at $23 \mathrm{GHz}$ and compare the resulting value to a prior. We use the difference between the extrapolated spectral index and the prior to augment the $\chi^{2}$ at each grid point,

$$
\chi^{2} \rightarrow \chi^{2}+\left(\frac{\beta_{23}-\beta_{p}}{\sigma_{p}}\right)^{2},
$$

where $\beta_{23}$ is the model spectral index evaluated at 23 $\mathrm{GHz}$, and $\beta_{p} \pm \sigma_{p}=-3.1 \pm 0.1$ is the prior at $23 \mathrm{GHz}$. The minimum $\chi^{2}$ over the entire grid then defines the best-fit model at that pixel. This allows inclusion of the spectral information derived from frequencies above 23 $\mathrm{GHz}$ without confusion from either additional emission components (thermal or spinning dust) above $23 \mathrm{GHz}$ or the missing zero level in the WMAP data.

Figure 4 shows the best-fit spectral parameter maps, while Figures 5 and 6 show the distribution of the bestfit values for the spectral index and curvature. Including the constraint at $23 \mathrm{GHz}$, the best-fit spectral index has mean value -2.64 and standard deviation 0.03 at reference frequency $\nu_{0}=310 \mathrm{MHz}$. The best-fit curvature has mean value -0.052 with standard deviation 0.005 . The corresponding spectral index at $23 \mathrm{GHz}$ is $\left\langle\beta_{23}\right\rangle=-3.09$ with standard deviation 0.05 . Inclusion of the prior at $23 \mathrm{GHz}$ does not induce a significant shift in the mean for the extrapolated spectral index, but does significantly reduce the pixel-to-pixel scatter.

Comparison of the parameter distributions with and without the spectral constraint at $23 \mathrm{GHz}$ demonstrates that the addition of the spectral constraint mainly affects the fitted curvature values (Figs. 5 and 6). The adopted value for the spectral constraint comes from independent analysis of WMAP data and is weighted toward regions of higher synchrotron intensity. We test whether this

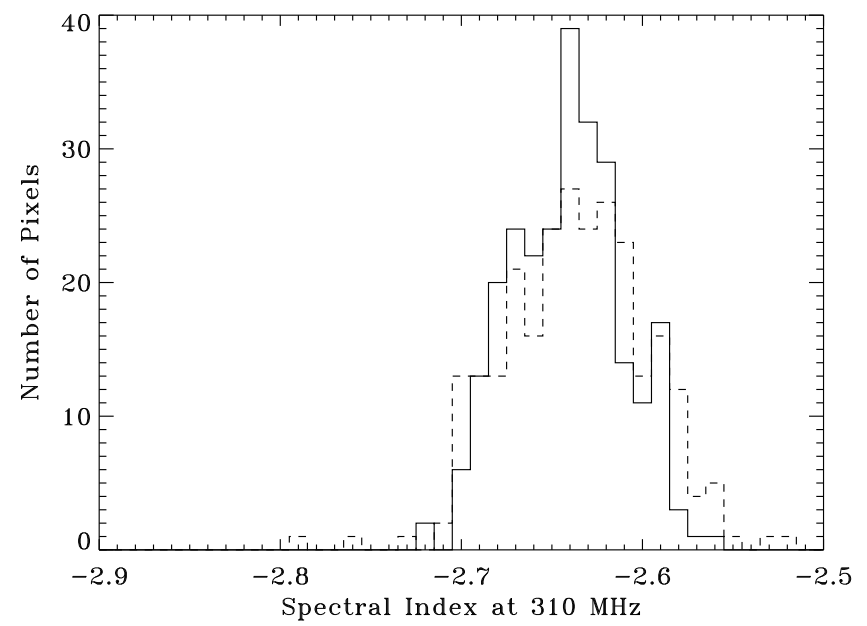

FIG. 5.- Spectral index evaluated at $310 \mathrm{MHz}$. The dashed line shows the distribution from the 10 -frequency radio data while the solid line includes the prior at $23 \mathrm{GHz}$.

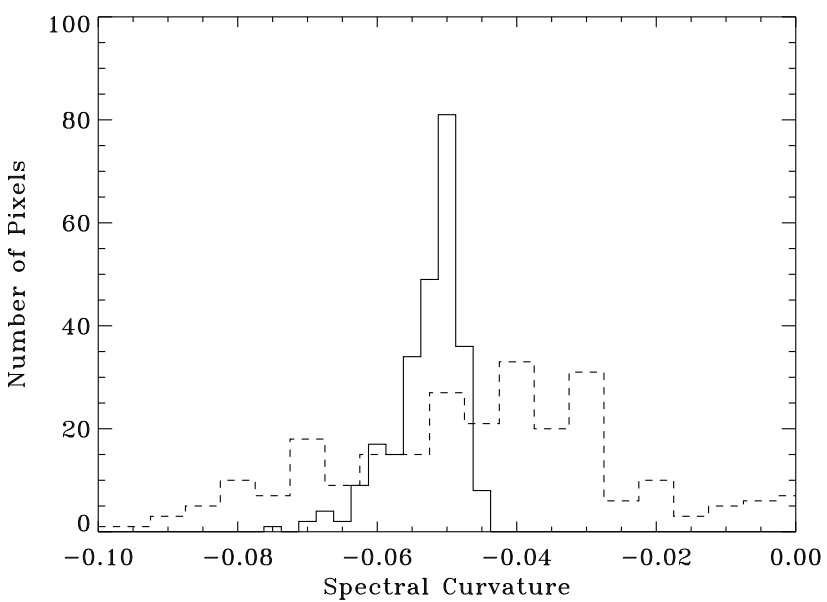

FIG. 6.- Spectral curvature at $310 \mathrm{MHz}$. The dashed line shows the distribution from the 10 -frequency radio data while the solid line includes the prior at $23 \mathrm{GHz}$. The mean curvature $C=-0.052$ corresponds to a steepening of the local spectral index by an amount $\Delta \beta=0.07$ every octave in frequency.

creates a bias in the fitted curvature values by splitting the observed sky coverage into two subsets of equal area, defined by the brightest and faintest $50 \%$ of the fitted amplitudes at $310 \mathrm{MHz}$. Within each subset, we compare the mean and standard deviation of the fitted curvature derived from the 10 -frequency fit or the enhanced fit including the constraint at $23 \mathrm{GHz}$. The "bright" subset shows no shift in the mean spectral curvature when the $23 \mathrm{GHz}$ constraint is added (although the scatter is significantly reduced). The "faint" subset shows both a reduction in scatter and a modest shift in the mean value, with curvature parameter steepening from -0.034 to -0.049 when the $23 \mathrm{GHz}$ constraint is included. This shift is less than one standard deviation: given the limited sky coverage, the available radio data do not yet provide significant evidence for spatial variation in the synchrotron curvature.

\section{DISCUSSION}

Radio data show statistically significant steepening of the synchrotron spectrum from $22 \mathrm{MHz}$ to $10 \mathrm{GHz}$. The nearly uniform relative uncertainty of the selected data minimizes dependence of the fitted parameters on offset or calibration errors at any one frequency. We test whether the best-fit parameters are particularly sensitive to any one input map by repeating the analysis after dropping one or two maps from the fit. We select either one radio survey $(22 \mathrm{MHz}, 45 \mathrm{MHz}, 408 \mathrm{MHz}$, or $1420 \mathrm{MHz}$ ) or a pair of ARCADE frequency channels $(3 \mathrm{GHz}, 8 \mathrm{GHz}$, or $10 \mathrm{GHz})$ and repeat the fit after deleting the corresponding elements from the data vector $T$ and covariance matrix $M$. The resulting shift in either the spectral index or curvature parameters is smaller than the pixel-to-pixel standard deviation using all 10 frequency channels. Systematic errors in the offset or temperature calibration do not appear to dominate the multi-frequency analysis.

The steepening of the synchrotron spectrum is broadly consistent with models of cosmic ray propagation in the Galactic magnetic field. Jaffe et al. (2011) combine ra- 
TABLE 2

Local Power-Law Spectral Index

\begin{tabular}{cc}
$\begin{array}{c}\text { Frequency } \\
\text { (GHz) }\end{array}$ & $\begin{array}{c}\text { Power-Law } \\
\text { Index }\end{array}$ \\
\hline 0.022 & -2.36 \\
0.045 & -2.44 \\
0.408 & -2.67 \\
3.3 & -2.89 \\
23 & -3.09 \\
33 & -3.13 \\
41 & -3.15 \\
61 & -3.19 \\
94 & -3.24 \\
\hline
\end{tabular}

dio observations with the GALPROF 3 cosmic ray propagation code to model synchrotron emission on the Galactic plane. They find a power-law index $-2.8<\beta<-2.74$ from $408 \mathrm{MHz}$ to $2.3 \mathrm{GHz}$ and $-2.98<\beta<-2.91$ from $2.3 \mathrm{GHz}$ to $23 \mathrm{GHz}$. The corresponding values for the spectral steepening model presented here are $\beta=-2.76$ from $408 \mathrm{MHz}$ to $2.3 \mathrm{GHz}$ and $\beta=-2.97$ from $2.3 \mathrm{GHz}$ to $23 \mathrm{GHz}$, in good agreement with the cosmic ray model.

We may use the best-fit values in each pixel to predict the synchrotron spectrum at higher frequencies where the emission is fainter and competing sources stronger. Previous attempts to disentangle competing emission from free-free, synchrotron, thermal dust, and spinning dust emission using WMAP data have suffered from degeneracy between the synchrotron and spinning dust emission, both of which are falling at frequencies above $33 \mathrm{GHz}$ (see, e.g., the discussion in Gold et al. (2011)). Extending the synchrotron curvature observed at lower frequencies into the millimeter band reduces confusion between the spinning dust and synchrotron spectra and may facilitate characterization of both the spatial distribution and frequency spectrum of spinning dust emission in the interstellar medium.

Table 2 shows the local power-law index $T \propto \nu^{\beta}$ at selected frequency bands. The modeled spectrum steepens by $\Delta \beta=0.07$ every octave in frequency, from $\beta=-2.67$ at $408 \mathrm{MHz}$ to $\beta=-3.24$ at $94 \mathrm{GHz}$. Note, however, that the spectral steepening observed at low frequencies can not continue indefinitely. Fermi measurements of the cosmic ray energy spectrum are consistent with a single power law from energy $7 \mathrm{GeV}$ to $1 \mathrm{TeV}$ (Abdo et al. 2009; Ackermann et al. 2010, 2012). If anything, the Fermi data suggest a modest flattening of the cosmic ray energy spectrum at higher energies, which would induce a positive curvature to the synchrotron spectrum at frequencies above $23 \mathrm{GHz}$.

Strong, Orlando, \& Jaffe (2011) combine radio data at $22,45,150,408$, and $1420 \mathrm{MHz}$ with WMAP data at 23 through $94 \mathrm{GHz}$ and Fermi Large Area Telescope cosmic ray measurements and the GALPROP code to estimate the magnetic field intensity and synchrotron spectrum from $22 \mathrm{MHz}$ to $94 \mathrm{GHz}$. Figure 7 compares the resulting synchrotron spectral index (diffusion model with injection index 1.3) to the curvature model from this paper. The cosmic ray model analyzes a limited latitude range $10^{\circ}<|b|<50^{\circ}$, which we follow in Figure 7 by excluding pixels at latitudes $|b|<10^{\circ}$. The two methods agree for frequencies below $408 \mathrm{MHz}$ (where they share common radio data) but differ at higher frequencies. The

\footnotetext{
3 http://galprop.stanford.edu
}

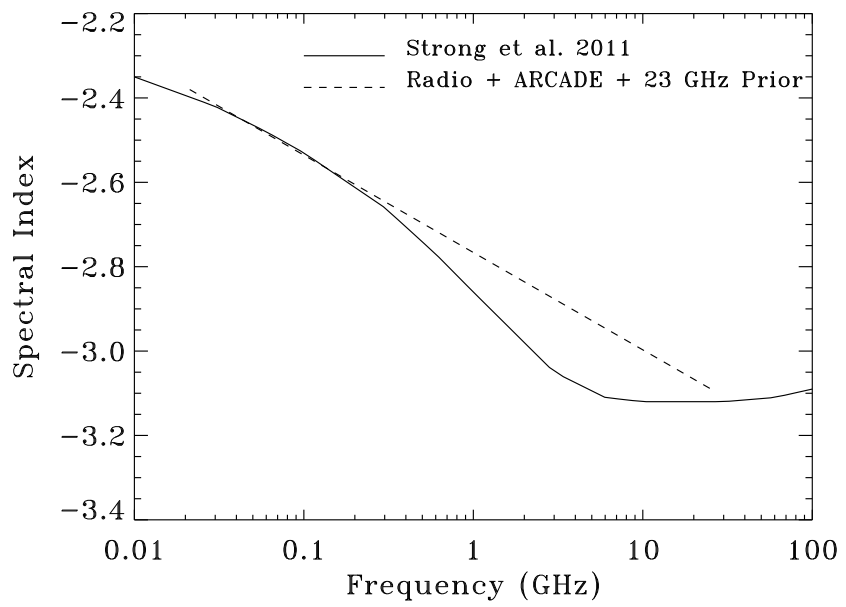

Fig. 7.- Comparison of the synchrotron spectral index as a function of frequency for different models. The break in the spectral index for the Strong. Orlando, \& Jaffe (2011) cosmic ray model at frequencies of a few $\mathrm{GHz}$ is not reproduced by the ARCADE 2 observations at $3-10 \mathrm{GHz}$, which prefer models with nearly constant spectral curvature.

cosmic ray model shows an increased synchrotron curvature from $408 \mathrm{MHz}$ to a few $\mathrm{GHz}$, followed by a spectral break to near-constant index $\beta \sim-3.1$ at higher frequencies. The ARCADE 2 data at $3-10 \mathrm{GHz}$ do not reproduce these features, but are instead consistent with constant spectral curvature from $22 \mathrm{MHz}$ to $10 \mathrm{GHz}$ (Fig 3 ).

Both models agree at frequencies near $23 \mathrm{GHz}$. The spectral index at $23 \mathrm{GHz}$ derived from the 10-frequency radio data without the $23 \mathrm{GHz}$ prior is consistent with independent measurements of the index above $23 \mathrm{GHz}$ and with the full radio fit including the $23 \mathrm{GHz}$ prior. The radio data, taken alone, do not support a break in the synchrotron spectrum at $\mathrm{GHz}$ frequencies. Comparison of the radio fit to the cosmic ray model suggests that any spectral break in the synchrotron spectrum must occur at frequencies above $23 \mathrm{GHz}$. Direct confirmation of the synchrotron spectrum above $23 \mathrm{GHz}$ remains a challenge.

\section{CONCLUSIONS}

Radio data are consistent with a synchrotron spectrum that steepens with frequency from $22 \mathrm{MHz}$ to $10 \mathrm{GHz}$. Direct comparison of low-frequency radio surveys with the WMAP data at 23 to $94 \mathrm{GHz}$ is complicated both by the presence of additional emission components at higher frequencies and by the subtraction of a substantial monopole component of sky emission by the differential WMAP instrument. The synchrotron spectral index at $23 \mathrm{GHz}$, derived using only lower-frequency radio surveys, is consistent with the value derived independently using only data at higher frequencies. We extend the radio data by comparing the extrapolated index at $23 \mathrm{GHz}$ to a prior based on higher-frequency data. The combined data have mean spectral index $\beta=-2.64 \pm 0.03$ and curvature $C=-0.052 \pm 0.005$ at reference frequency 0.31 $\mathrm{GHz}$. The measured spectrum steepens by an amount $\Delta \beta=0.07$ every octave in frequency. Comparison of the radio data to models including the cosmic ray en- 
ergy spectrum suggests that any break in the synchrotron spectrum must occur at frequencies above $23 \mathrm{GHz}$.

This research is based upon work supported by the
National Aeronautics and Space Administration through the Science Mission Directorate under the Astronomy and Physics Research and Analysis suborbital program.

\section{REFERENCES}

Abdo, A. A., et al., 2009, Phys. Rev. Lett., 102, 181101

Ackermann, M., et al., 2012, Phys. Rev. Lett., 108, 011103

Ackermann, M., et al., 2010, Phys. Rev. D, 82, 092004

Ali-Haïmoud, Y. Hirata, C. M. \& Dickinson, C., 2009, MNRAS, 395,1055

Alvarez, H., Aparici, J., May, J., and Olmos, F., 1997, A\&AS, 124,315

Bennett, C. L., et al., 2003, ApJS, 148, 97

Dobler, G. and Finkbeiner, D. P., 2008, ApJ, 680, 1222

Draine, B. T. and Lazarian, A., 1998, ApJ, 494, L19

Dunkley, J, et al. 2009, ApJ, 701, 1804

Gold, B. et al., 2011, ApJS, 192, 15

Haslam, C. G. T., et al., 1981, A\&A, 100, 209

Hinshaw, G., et al., 2009, ApJS, 180, 225

Hoang, T., Draine, B. T., \& Lazarian, A., 2010, ApJ, 715, 1462

Jaffe, T. R., et al., 2011, MNRAS, 416, 1152

Kogut, A. et al., 2011, ApJ, 734, 4

Kogut, A. et al., 2004, ApJS, 154, 493

Kogut, A., et al., 1996, ApJ, 460, 1

Maeda, K., Alvarez, H., Aparici, J., May, J., and Reich, P., 1999, A\&AS, 140, 145
Miville-Deschênes, M.-A., et al., 2008, A\&A, 490, 1093

de Oliveira-Costa, A., et al., 2004, ApJ, 606, L89

de Oliveira-Costa, A., et al., 1997, ApJ, 482, L17

Oster, L., 1961, Phys. Rev., 121, 961

Planck Collaboration, 2011, A\&A, 536, A20

Reich, P., Testori, J. C., and Reich, W., 2001, A\&A, 376, 861

Reich, P., and Reich, W., 1986, A\&AS, 63, 205

Roger, R. S., Costain, C. H., Landecker, T. L., and Swerdlyk, C. M., 1999, A\&AS, 137, 7

Rybicki, G. B. \& Lightman, A. 1979, Radiative Processes in Astrophysics (Wiley \& Sons: New York)

Schwinger, J., 1949, Phys. Rev., 75, 1912

Singal, J. et al., 2011, ApJ, 730, 138

Strong, A. W., Moskalenko, I. V., \& Ptuskin, V. S., 2007, Annu. Rev. Nucl. Part. Sci., 57, 285

Strong, A. W., Orlando, E., \& Jaffe, T. R., 2011, A\&A, 534, A54

Westfold, K. C., 1959, ApJ, 130, 241

Ysard, N. \& Verstraete, L., 2010, A\&A, 509, A12

Ysard, N., Miville-Deschênes, M. A., \& Verstraete, L., 2010, A\&A, 509, L1 